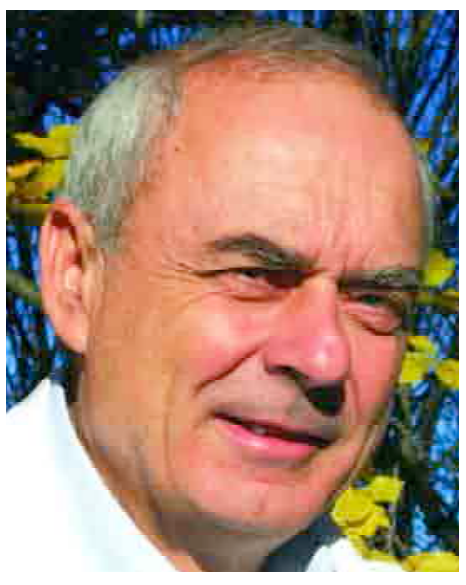

\title{
Interview mit Dr. med. Alain Barmont, Oberarzt Physikalische Therapie und Rehabilitation am Spital Beau-Séjour, Universitätsspital Genf (HUG) \\ Assalix ${ }^{\circledR}-$ ein Weidenrinden-Extrakt
}

Während akute Schmerzzustände als Folge von Traumata oder Überbeanspruchung sehr effizient mit nichtsteroidalen Antirheumatika behandelt werden können, muss man bei chronischen rheumatischen Beschwerden neben der zuverlässigen Wirksamkeit auch eine langfristig gute Verträglichkeit fordern. Diesem Anforderungsprofil entspricht das Weidenrindenextrakt-Präparat Assalix ${ }^{\circledR}$, das seit Mai 2003 in der Schweiz für die Behandlung von rheumatischen Beschwerden wie z.B. Kreuzschmerzen, Rücken- und Nackenschmerzen zugelassen und kassenpflichtig ist.

\section{Was ist Assalix ${ }^{\circledR}$ ?}

Dr. Barmont: Assalix ${ }^{\circledR}$ ist ein Präparat, das gegen Entzündung und Schmerz wirkt und daher Patienten mit chronischen Schmerzen eine bessere Lebensqualität ermöglicht. Bei Assalix ${ }^{\circledR}$ handelt es sich um einen WeidenrindenExtrakt, der Salicin enthält, welches zu Salicylsäure metabolisiert wird, jedoch in einer 60-mal geringeren Konzentration als beim Aspirin ${ }^{\circledR}$. Da es sich um einen pflanzlichen Extrakt und nicht um ein Synthetikum handelt, finden sich in Assalix ${ }^{\circledR}$ noch andere aktive Substanzen (vor allem SalicinDerivate, Catechine und Procyanidine). Genau wie die herkömmlichen nicht steroidalen Antirheumatika (NSAR) greift dieses neue Medikament in die wichtigsten Entzündungsvorgänge ein. Unter anderem hemmt Assalix ${ }^{\circledR}$ die Enzyme Cyclooxygenase (COX) und Lipooxygenase und vermindert so die Synthese von Prostaglandinen und Leukotrienen [1].
Wozu wird ein Produkt wie Assalix ${ }^{\circledR}$ entwickelt, wenn es bereits NSAR zur Behandlungen von Entzündungen gibt?

Dr. Barmont: Weil die NSAR Nebenwirkungen haben, die nicht zu vernachlässigen sind. Prostaglandine fördern nicht nur Entzündungsvorgänge, sondern sie dienen auch dem Schutz der Magenschleimhaut und sie sind an physiologischen Prozessen von Niere und Thrombozyten beteiligt. Durch die Cyclooxygenase-Hemmung besteht die Gefahr, Gastritiden und Ulzerationen im Verdauungstrakt hervorzurufen. Die länger dauernde Einnahme von NSAR kann zu Blutungen und zu einer Perforation der Magenwand führen. Auch wenn das Risiko für den Einzelnen eher gering ist, ist doch die Anzahl der Patienten, die NSAR einnehmen, so gross, dass die Risiken nicht vernachlässigt werden dürfen. Aus genau diesem Grund wurden ja auch die neuen entzündungshemmen- 
den selektiven COX-2-Inhibitoren wie Vioxx ${ }^{\circledR}$ entwickelt. Es hatte sich nämlich gezeigt, dass es zwei Varianten der Cyclooxygenase gibt und dass nur die COX-2 bei Entzündungen mitbeteiligt ist, während die COX-1 vor allem günstige Wirkungen im Körper entfaltet. Diese Therapiestrategie funktionierte bis anhin gut. Allerdings musste Vioxx ${ }^{\circledR}$ kürzlich vom Markt zurückgezogen werden, weil es die Wahrscheinlichkeit erhöht, dass ein Herzinfarkt oder ein zerebrovaskulärer Insult auftritt - eine unerwünschte Wirkung, die bei den ersten klinischen Studien nicht erkannt wurde. Zudem ist nicht klar, was mit den anderen COX-2-Hemmern geschieht, die noch auf dem Markt sind.

\section{Was hat denn Assalix ${ }^{\circledR}$ mit all dem zu tun?}

Dr. Barmont: Nun, Assalix ${ }^{\circledR}$ weist alle diese Nebenwirkungen eben nicht auf. Weil es nicht auf die COX-1 einwirkt und nur eine sehr geringe Menge Salicylsäure gebildet wird, wird es sehr gut vertragen, sowohl was den Verdauungstrakt betrifft, wie auch betreffend Gerinnung und Nierenfunktion. Selbstverständlich sollte es aus genau diesem Grund weder zusammen mit Antikoagulanzien verschrieben, noch in Kombination mit Aspirin $^{\circledR}$ verabreicht werden.
Wie gut ist seine Wirksamkeit gegen Schmerzen? Könnte es die NSAR ersetzen?

Dr. Barmont: Es konnte gezeigt werden, dass Assalix ${ }^{\circledR}$ Schmerz und Entzündungsvorgänge mindert, aber wie stark seine Wirkung im Vergleich zu den herkömmlichen Medikamenten ist, ist noch nicht bekannt. Die alten Medikamente wird es nicht ganz ersetzen können, vor allem dann nicht, wenn stärkste Schmerzen vorliegen, aber man kann es vom Beginn der Behandlung an in Kombination mit diesen Medikamenten verabreichen. Zudem hat eine Studie gezeigt, dass die Patienten deutlich weniger NSAR einnehmen, wenn ihnen gleichzeitig Assalix $^{\circledR}$ verabreicht wird.

Welche persönlichen Erfahrungen haben Sie mit diesem neuen Produkt gemacht?

Dr. Barmont: Die Patienten, die zu mir kommen, leiden an Beschwerden des Bewegungsapparates, vor allem an Arthrose und an Kreuzschmerzen. Meine spezialärztliche Tätigkeit ist die physikalische Medizin. Bevor ich Patienten Medikamente gebe, manipuliere ich. Doch immer häufiger verordne ich Assalix ${ }^{\circledR}$ anstelle von NSAR und bin dann auch zufrieden damit. Auf jeden Fall bitten mich die Patienten nicht, ihnen wieder ihr altes Medikament zu geben.

Meine Erfahrungen bestärken mich in meiner Meinung, dass Assalix ${ }^{\circledR}$ seinen Platz in der Rheumatherapie hat. Dies ist zum Beispiel bei Patienten der Fall, die ihren ersten Arthroseschub erleiden und noch nie NSAR eingenommen haben. Anders verhält es sich bei Personen, die seit Jahren an chronischen Schmerzen leiden. Man kann ihnen nicht über die ganze Zeit hinweg NSAR verschreiben. Meistens gibt man dann ein Schmerzmittel wie Dafalgan ${ }^{\circledR}$. Assalix $^{\circledR}$ erlaubt es nun aber, die Gabe von NSAR für eine Zeitlang zu unterbrechen, da es nicht nur eine schmerzlindernde Wirkung hat, sondern gleichzeitig auch noch gegen die Entzündung wirkt, was ausgesprochen von Vorteil ist.

\section{Herr Dr. Barmont, wir danken Ihnen} für das interessante Gespräch!

Interview: Dr. Stefan R. Becker, Basel

\section{Literatur}

1. März, R.W. und F. Kemper: Weidenrindenextrakt - Wirkungen und Wirksamkeit. Erkenntnisstand zu Pharmakologie, Toxikologie und Klinik. Wien Med Wschr 2002. 152: S. 354-359.
Gekürzte Fachinformation Assalix ${ }^{\circledR}$
(Trockenextrakt aus Weidenrinde).
Indikationen: Rheumatische Be-
schwerden wie Kreuzschmerzen,
Rücken- und Nackenschmerzen.
Dosierung: 1-2 mal täglich 1 Dragée.
Anwendungseinschränkungen:
Überempfindlichkeit gegen Salicylate.
Unerwünschte Wrkungen: Über-
empfindlichkeitsrektionen (selten).
Packungen: Dragées $20^{*}, 50 *, 100 *$.
Verkaufskategorie D. Ausführliche
Angaben siehe Arzneimittel-Kompen-
dium der Schweiz. *kassenpflichtig 